\title{
Catamenial pneumothorax: a case report of recurrent spontaneous pneumothorax
}

\author{
Timuçin Alar ${ }^{1 a}$, Ahmet Uysal'², İsmail Ertuğrul Gedik \\ 'Department of Thoracic Surgery, Faculty of Medicine, Çanakkale Onsekiz Mart University, Çanakkale, Turkey \\ ${ }^{2}$ Department of Obstetrics and Gynecology, Faculty of Medicine, Çanakkale Onsekiz Mart University, Çanakkale, Turkey
}

\begin{abstract}
Catamenial pneumothorax $(\mathrm{CP})$ is a type of recurrent pneumothorax that occurs during menstrual periods in women. CP is a special type of pneumothorax related with the menstrual period and the treatment consists of both medical and surgical methods. First line of surgical treatment is exploration of thorax via VATS and revealing the pathophysiological factors of CP. Despite the surgical treatment techniques, a postoperative recurrence rate of $\mathrm{CP}$ is $30 \%$. We present a recurrent pneumothorax case who had 5 pneumothorax episodes and diagnosed as CP. A left VATS apical wedge resection and pleurectomy was performed and oral contraceptive was administered. and she did not have a recurrent pneumothorax episode for last 2 years.
\end{abstract}

Key Words: Pneumothorax; catamenial pneumothorax; endometriosis

Corresponding Authora: Timuçin Alar, MD. Department of Thoracic Surgery, Faculty of Medicine, Çanakkale Onsekiz Mart University, Çanakkale, Turkey.

E-mail: timalar@yahoo.com

Received 21.06.2016 accepted 05.11.2016 


\section{Introduction}

Catamenial pneumothorax (CP) which is derived from the Greek word catamenial meaning monthly. $\mathrm{CP}$ is a type of recurrent pneumothorax that occurs during menstrual periods in women of breeding age [1]. CP is a pneumothorax that starts within a time period of 24 hours before and 72 hours after the onset of a menstrual period [2]. The mean age of patients with $\mathrm{CP}$ is 35 years (range 15-54) [3]. It should be noted that thorax is the most common site of extra-pelvic endometriosis which is believed to be the primary etiological factor in CP.

We present a recurrent pneumothorax case who had 5 pneumothorax episodes and one of them is during the postoperative period, diagnosed as $\mathrm{CP}$.

\section{Case Report}

A 28-year-old female patient presented to our hospital's emergency department with the chief complaint of left sided chest pain. Patient's history revealed that the pain started abruptly 4 hours before her admission and is aggravated by inspiration. Her history also revealed that she was in her menstrual period. She mentioned that she had similar left sided chest pain started during menstrual cycles twice last year. She admitted to other hospitals for these episodes and was treated with nasal oxygen inhalation therapy. This episode of pneumothorax was considered as the first episode because we couldn't obtain her past medical record. Physical examination revealed diminished left apical lung auscultation sounds. Left sided spontaneous pneumothorax was found in posterior-anterior (PA) chest x-ray (Figure 1).

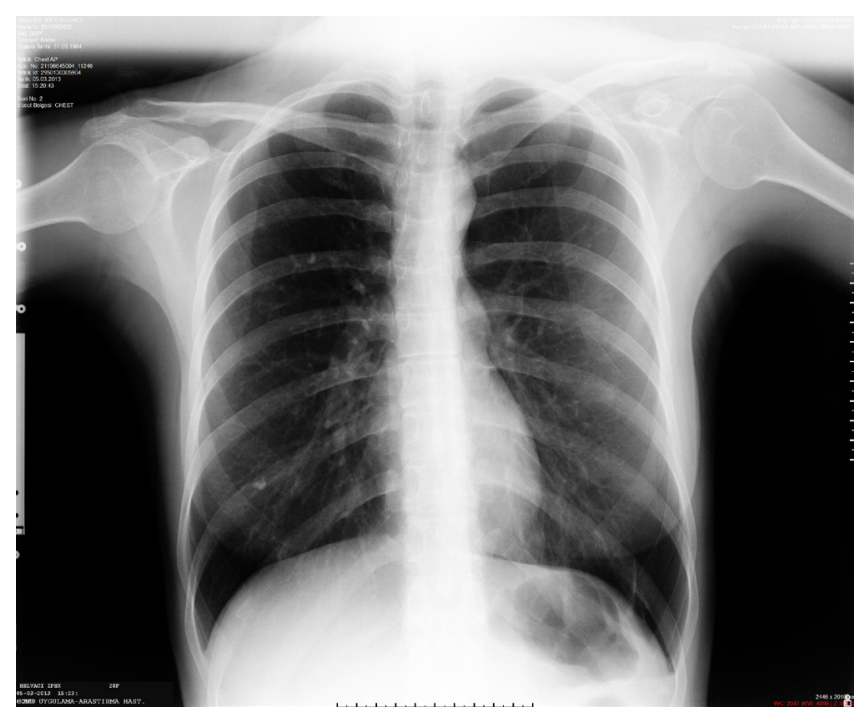

Figure 1. Postero-anterior chest x-ray of the patient in emergency service
Patient was hospitalized and nasal oxygen was administered at $3 \mathrm{lt} / \mathrm{min}$. Her pleuritic chest pain diminished and lung auscultation sounds were normal on follow-up. PA chest x-ray control revealed left lung was completely expanded and no pneumothorax was found. She was discharged on her fourth day. Her control examination 1 week later was normal. She re-admitted to our outpatient clinics approximately 3 months after her first admission with similar complaints. Her history revealed that her complaints started during menstrual period. Physical examination revealed diminished left apical lung auscultation sounds. Left sided recurrent spontaneous pneumothorax was found in PA chest x-ray. She was hospitalized again and left VATS apical wedge resection and left apical pleurectomy was performed. Neither diaphragmatic pores nor lesions compatible with thoracic endometriosis in left hemi-thorax were found during the operation. Multiple biopsies from the parietal pleura of the left hemi-thorax were performed in order to find thoracic endometriosis. Pathologic examination did not reveal endometriosis. Apical wedge resection material was also examined by pathology department and reported as bullous emphysema. Air drainage has stopped at the fourth postoperative day. Physical examination and the PA chest $\mathrm{x}$-ray was normal. Thoracic drain was removed at the same day. Patient was consulted to the gynecology clinics and oral contraceptive including 2 $\mathrm{mg}$ of dienogest was administered. She was discharged at the fifth postoperative day. Her control examination 1 week later was normal (Figure 2).

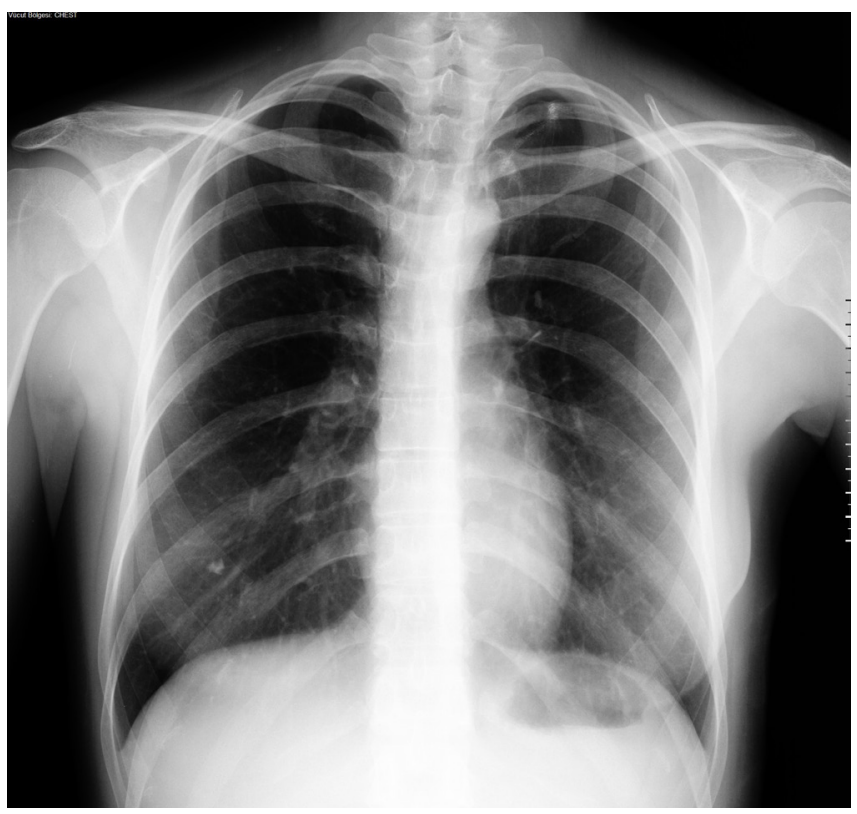

Figure 2. Post-operative postero-anterior chest x-ray of the patient 1 week later 
She re-admitted to our hospital's emergency department clinics approximately 2 months after her first admission with same complaints. Her medical history revealed that she stopped taking the oral contraceptives willingly because she was planning for pregnancy. Physical examination revealed diminished left apical lung auscultation sounds. Left sided recurrent spontaneous pneumothorax was found in PA chest $x$-ray. Patient was hospitalized and nasal oxygen was administered at $31 \mathrm{t} / \mathrm{min}$. Patient was consulted to the gynecology clinics and was administered oral contraceptives including $2 \mathrm{mg}$ of cyproterone acetate and $0,035 \mathrm{mg}$ of ethinyl estradiol. Physical examination and the PA chest $x$-ray at the fourth day of hospitalization was normal. She was discharged at the same day. Oral contraceptive therapy continued for 6 months because of recurrent pneumothorax. The patient is in her follow-up period for almost 2 years and she did not have a recurrent pneumothorax episode.

\section{Discussion}

$\mathrm{CP}$ is a special type of pneumothorax related with the menstrual period that can only be seen in women because of its pathophysiological mechanism and is usually misdiagnosed. There are two etiological factors that are blamed in the pathophysiology of CP. First one is thoracic endometriosis and the second is single or multiple diaphragmatic pores. Our patient was infertile which supported the diagnosis of endometriosis but we could not find any endometrial tissue within the thorax or pores on the diaphragm during the videothoracoscopic examination. Pathological examination of the pleural biopsies that were obtained during the surgery did not reveal any endometriosis or diaphragmatic pores despite the fact that diaphragmatic pores can be seen in $23-88 \%$ of the patients and endometriosis usually accompanies with these pores [4]. The treatment of CP consists of both medical and surgical treatment. First line of surgical treatment is exploration of thorax via VATS and revealing the pathophysiological factors of CP. It consists of wedge resection of lung parenchyma that includes the endometriotic tissue and pleurectomy for pleural endometriosis. Diaphragmatic defects should be sutured if present and if there are numerous pores that cannot be repaired by suturing, surgeon should perform partial or total diaphragmatic resection and reconstruction. Despite these surgical treatment techniques postoperative recurrence rates of $\mathrm{CP}$ is $30 \%$ [5]. Our patient had a recurrent episode of CP 2 months after surgery despite that she had undergone videothoracoscopic apical wedge resection and apical pleurectomy. Because of high incidences of postoperative recurrence, medical treatment should be combined with the surgi- cal treatment. Gonadotropic hormone analogues should be administered to the patients for 3-6 months in order to allow the pleurodesis to take effect [6]. Our patient had a recurrent episode of $\mathrm{CP}$ after she stopped taking her medical treatment that only lasted for a month. This shows the importance of medical treatment in CP. Our patient had no recurrences for 2 years after she completed her medical treatment that lasted 6 months. It is tenable that the combination of medical with surgical treatment prevents recurrences in CP. But proper history taking as in our case prevents unnecessary surgical procedures and helps in selecting the optimum medical treatment strategy.

In conclusion, $\mathrm{CP}$ causes recurrences even after the surgery. Medical treatment is successful in preventing the recurrences. Physicians should question that if the patients are taking their medical treatment regularly before deciding that the surgical treatment failed in recurrent pneumothorax.

\section{Declaration of conflicting interests}

The author declared no conflicts of interest with respect to the authorship and/or publication of this article.

\section{Funding}

The author received no financial support for the research and/or authorship of this article.

\section{References}

1. Visouli AN, Zarogoulidis K, Kougioumtzi I, Huang $\mathrm{H}, \mathrm{Li}$ Q, Dryllis G, et al. Catamenial pneumothorax. J Thorac Dis 2014; 6: S448-60.

2. Baoquan L, Liangjian Z, Qiang W, Hai J, Hezhong C, Zhiyun X. Catamenial pneumothorax associated with multiple diaphragmatic perforations and pneumoperitoneum in a reproductive woman. J Formos Med Assoc 2014; 113: 385-7.

3. Alifano M, Trisolini R, Cancellieri A, Regnard JF. Thoracic endometriosis: current knowledge. Ann Thorac Surg 2006; 81: 761-9.

4. Joseph J, Sahn SA. Thoracic endometriosis syndrome: new observations from an analysis of 110 cases. Am J Med 1996; 100: 164-70.

5. Baysungur V, Tezel C, Okur E, Yilmaz B. Recurrent pneumothorax diagnosed as catamenial after videothoracoscopic examination of the pleural cavity. Surg Laparosc Endosc Percutan Tech 2011; 21: 81-3.

6. Leong AC, Coonar AS, Lang-Lazdunski L. Catamenial pneumothorax: surgical repair of the diaphragm and hormone treatment. Ann R Coll Surg 2006; 88: 547-9. 